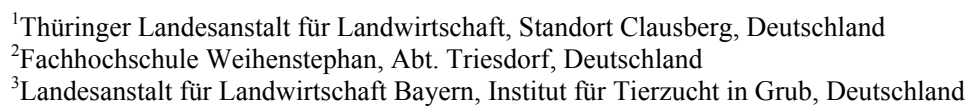

\title{
Futteraufnahme und Fressverhalten wachsender Lämmer 1. Mitteilung: Heritabilitäten und genetische Korrelationen
}

\begin{abstract}
Title: Feed intake and eating behaviour of growing lambs, $1^{\text {st }}$ communication: heritabilities and genetic correlations

In the test stations "Grub" (Bavaria) and "Schöndorf" (Thuringia) data were collected on lambs of the breeds Merinolandschaf and Merino Longwool. Feed intake during the whole test period (FAP), feed efficiency (FAW), daily feed intake (TFM), the number of daily visits (TBH) and eating time (TBD) of lambs were measured. In total, data from 1,608 lambs with 37,161 daily records in "Grub" and 1,424 lambs with 35,356 daily records in "Schöndorf" were included. The aim of the present study was to estimate heritabilities and genetic correlations for different traits of feed intake and eating behaviour. In "Schöndorf" the heritability of FAP and FAW was 0.29 and 0.32 , respectively. In the test station "Grub" the heritability of the same traits was 0.58 and 0.57 , respectively. The heritability of TBH, TBD and TFM were $0.33,0.25$ and 0.05 , respectively, in "Schöndorf" and $0.49,0.36$ and 0.10 , respectively, in "Grub". The genetic correlations between the eating behaviour traits TBH and TBD on one hand and feed intake (FAP, FAW, TFM) on the other hand were low.

It could be concluded that feed intake as well as eating behaviour showed considerable variances and heritabilities. Further, eating behaviour was not a good indicator of feed intake.
\end{abstract}

Keywords: sheep breeding, feed intake, feed efficiency, eating behaviour, heritability, genetic correlations

\section{Zusammenfassung}

In den Leistungsprüfstationen Grub (Bayern) und Schöndorf (Thüringen) wurde die Futteraufnahme im Prüfzeitraum (FAP), der Futteraufwand (FAW), die täglich aufgenommene Futtermenge (TFM), die tägliche Besuchshäufigkeit (TBH) und die tägliche Besuchsdauer in der Futterstation (TBD) an Lämmern der Rassen Merinoland- und Merinolangwollschaf erfasst. Insgesamt konnten 1.608 Lämmer mit 37.161 Tagesdatensätzen aus Grub und 1.424 Lämmer mit 35.356 Tagesdatensätzen aus Schöndorf einbezogen werden. Das Ziel der Untersuchung bestand in der Schätzung der Heritabilitäten und der genetischen Korrelationen der erfassten Merkmale.

Die Heritabilität betrug für FAP und FAW in Schöndorf 0,29 bzw. 0,32 und in Grub 0,58 bzw. 0,57. Für die Merkmale TBH, TBD und TFM konnten an Schöndorfer Daten Erblichkeitsgrade von 0,33, 0,25 und 0,05 sowie für die Prüfung in Grub Werte von 0,49, 0,36 und 0,10 geschätzt werden. Die genetischen Korrelationen zwischen den Verhaltensmerkmalen TBH und TBD einerseits und der Futteraufnahme (FAP, FAW, TFM) andererseits waren niedrig.

Es bleibt festzuhalten, dass sowohl die Futteraufnahme als auch das Fressverhalten ausreichende Varianzen und Heritabilitäten aufwiesen. Aus dem Fressverhalten konnte nicht auf die aufgenommene Futtermenge geschlossen werden.

Schlüsselwörter: Schafzucht, Futteraufnahme, Futterverwertung, Fressverhalten, Heritabilität, genetische Korrelationen

\section{Einleitung}

In der Lämmermast hat eine effiziente Verwertung des aufgenommenen Futters eine große wirtschaftliche Bedeutung. Tiere, die weniger Futter für ein Kilogramm Gewichtszuwachs benötigen als andere, verursachen geringere Futterkosten bei gleicher Leistung. 
Allerdings setzt eine Einbeziehung des Futteraufwandes in die praktische Zuchtarbeit voraus, dass keine negative Beziehung zur Futteraufnahme vorhanden ist. Letzteres ist ein wichtiges Kriterium bei Mutterschafen, die selten mit hochkonzentrierten Futtermitteln wie z. B. Kraftfutter sondern meist mit natürlichem Aufwuchs marginaler Grünlandstandorte ihren Nährstoffbedarf decken müssen. Außerdem liegen nur wenige Erkenntnisse zur Varianz und Heritabilität des Futteraufwandes und der Futteraufnahme vor. Dies gilt auch für die entsprechenden Verhaltensmerkmale, die in Verbindung mit der Futteraufnahme stehen. Weiterhin sind die genetischen Beziehungen zwischen den Verhaltensmerkmalen und den Merkmalen der Futteraufnahme zu analysieren, um die Selektionswürdigkeit der einzelnen Merkmale beurteilen zu können.

Vergleichsweise intensiv erforscht wurden Merkmale der Futteraufnahme bei Rindern. Allerdings sind diese nicht ohne Vorbehalte auf Schafe übertragbar. So zielten z. B. Analysen an Milchrindbullen auf die Verbesserung der Gesundheit ihrer Töchter, die besonders zu Beginn der Laktation ein Energiedefizit aufweisen (WASSMUTH, 1999; POTTHAST, 1999).

Das Ziel der vorliegenden Untersuchung bildete die Schätzung von Heritabilitäten und genetischen Korrelationen für die Futteraufnahme sowie das Futteraufnahmeverhalten.

\section{Material und Methoden}

\section{Datenmaterial und Tiere}

In den Leistungsprüfstationen Schöndorf (Thüringer Lehr-, Prüf- und Versuchsgut Buttelstedt $\mathrm{GmbH}$ ) und Grub (Landesanstalt für Landwirtschaft) standen Futterautomaten zur Verfügung, mit denen die Besuchsanzahl und -dauer der Lämmer in den Kraftfutterstationen sowie die aufgenommene Futtermenge erfasst wurde. Besuche der Station ohne Futteraufnahme blieben dabei unberücksichtigt. Besuche über Mitternacht rechnete man dem Tag zu, an dem sie begannen. Während in Schöndorf sowohl Merinolandschafe als auch Merinolangwollschafe einbezogen wurden, waren es in Grub ausschließlich Merinolandschafe. Die Prüfung erfolgte im Gewichtsbereich 20 bis $42 \mathrm{~kg}$.

Für den gesamten Prüfzeitraum wurden folgende Merkmale berechnet:

FAP (kg TM) - Futteraufnahme in Kilogramm Trockenmasse

FAW (kg TM) - Futteraufwand gemessen als aufgenommene Futtermenge in

Kilogramm Trockenmasse pro Kilogramm Lebendgewichtszuwachs pro Lamm.

Während in Grub Daten seit 2002 bis 2006 einbezogen wurden, stammen die Thüringer Werte aus dem Zeitraum 2004 bis 2006.

Einen Überblick über die statistischen Maßzahlen zeigt Tabelle 1. Die berechneten Variationskoeffizienten erreichen Größenordnungen, die die Merkmale als selektionswürdig erscheinen lässt.

Folgende Tageswerte wurden aus den einzelnen Datensätzen der Kraftfutterstationen für jedes Lamm berechnet:

TBH (n/Tag) - tägliche Besuchshäufigkeit in der Kraftfutterstation

TBD (min/Tag) - tägliche Besuchsdauer in der Kraftfutterstation

TFM (kg/Tag) - täglich aufgenommene Futtermenge. 
Tabelle 1

Anzahl Tiere (n), Rohmittelwert ( $\overline{\mathrm{x}}$ ), Standardabweichung (S), Minimum (Min), Maximum (Max) für Merkmale der Futteraufnahme (FAP) und des -aufwandes (FAW) im gesamten Prüfzeitraum in Schöndorf und Grub (Number of tested lambs [n], mean $[\overline{\mathrm{x}}]$, phenotypic standard deviation [S], minimum [Min], maximum $[\mathrm{Max}]$ for feed intake [FAP] and feed efficiency [FAW] in the whole test period in "Schöndorf" and "Grub")

\begin{tabular}{lrrrrrr}
\hline Merkmal & $\mathrm{N}$ & Min & Max & $\overline{\mathrm{x}}$ & $\mathrm{S}$ & VK (\%) \\
\hline Schöndorf & & & & & & \\
FAP & 1.424 & 4,6 & 91,6 & 50,6 & 10,8 & 21,3 \\
FAW & 1.424 & 1,8 & 4,4 & 3,0 & 0,3 & 10,0 \\
Grub & & & & & & \\
FAP & 1.608 & 28,1 & 90,6 & 59,5 & 9,2 & 15,5 \\
FAW & 1.720 & 2,9 & 6,3 & 4,0 & 0,4 & 10,0 \\
\hline
\end{tabular}

Tabelle 2 gibt einen Überblick über die statistischen Maßzahlen. Es wird deutlich, dass auch die Merkmale des Futteraufnahmeverhaltens züchterisch interessante Variationskoeffizienten aufweisen.

Tabelle 2

Anzahl Tiere (n), Rohmittelwert ( $\bar{x}$ ), Standardabweichung (S), Minimum (Min), Maximum (Max) für die tägliche Besuchshäufigkeit (TBH), die tägliche Besuchsdauer (TBD) und die täglich aufgenommene Futtermenge (TFM) in Schöndorf und Grub

(Number of tested lambs [n], mean [x], phenotypic standard deviation [S], minimum [Min], maximum [Max] for daily visits at the feeding rack [TBH], daily eating time [TBD] and daily feed intake [TFM] in "Schöndorf" and "Grub")

\begin{tabular}{lrrrr}
\hline Merkmal & $\mathrm{N}$ & $\overline{\mathrm{x}}$ & $\mathrm{S}$ & $\mathrm{VK}(\%)$ \\
\hline Schöndorf & & & & \\
TBH & 35.321 & 10,5 & 4,4 & 41,9 \\
TBD & 35.356 & 60,3 & 18,7 & 31,0 \\
TFM & 35.356 & 1,2 & 0,3 & 25,0 \\
Grub & & & & \\
TBH & 37.161 & 20,2 & 11,0 & 54,5 \\
TBD & 37.134 & 79,0 & 25,5 & 32,3 \\
TFM & 37.160 & 1,3 & 0,4 & 30,8 \\
\hline
\end{tabular}

Die statistischen Maßzahlen in den Tabellen 1 und 2 machen prinzipielle Unterschiede der Futterdaten aus beiden Stationen deutlich. Dabei sind die Unterschiede in den Verhaltensmerkmalen wesentlich größer als bei der Futtermenge. Als eine Ursache können die verschiedenen Größen der Haltungsgruppen gelten. Während sich in Schöndorf 10 Lämmer einer Gruppe einen Automaten teilen mussten, waren dies in Grub nur 4-5. Diese Unterschiede zwischen den Stationen machten eine getrennte Auswertung der Daten der verschiedenen Stationen erforderlich.

\section{Methoden}

Die Varianzkomponenten wurden mit dem Programmpaket VCE, Version 5.1 (KOVAC et al., 2003) bei Einsatz des analytischen Gradienten geschätzt.

Für die genetischen Korrelationen kamen multivariate Schätzläufe zum Einsatz. Für die Futteraufnahme (FAP) und den Futteraufwand (FAW) kam folgendes Modell zur Anwendung:

$$
\mathrm{y}_{\mathrm{ijklm}}=\mu+\mathrm{HJ}_{\mathrm{i}}+\mathrm{R}_{\mathrm{j}}+\mathrm{EQ}_{\mathrm{k}}+\mathrm{b}_{1} \times \mathrm{KPB}_{\mathrm{ijkl}}+\mathrm{b}_{2} \times \mathrm{KPE}_{\mathrm{ijkl}} \mathrm{a}_{1}+\mathrm{e}_{\mathrm{ijklm}}
$$

mit: $\mathrm{y}_{\mathrm{ijklm}}=$ Beobachtungswerte des 1-ten Tieres; $\mathrm{HJ}_{\mathrm{i}}=$ fixer Effekt des i-ten Herkunftsbetriebsjahres, $R_{j}=$ fixer Effekt der $\mathrm{j}$-ten Rasse $(\mathrm{ML}, \mathrm{MLW}), \mathrm{EQ}_{\mathrm{k}}=$ fixer Effekt des k-ten Einstallungsquartals, $b=$ lineare Regressionskoeffizienten, $\mathrm{KPB}_{\mathrm{ijk} \mathrm{l}}=$ Körpermasse des 1-ten Tieres zu Prüfbeginn, $\mathrm{KPE}_{\mathrm{ijkl}}=$ Körpermasse des 1-ten Tieres zu Prüfende, $\mathrm{a}_{1}=$ additiv genetischer Effekt des Tieres $1, \mathrm{pu}_{1}=$ permanenter Umwelteffekt des Tieres $1, \mathrm{e}_{\mathrm{ijklm}}=$ zufälliger Restfehler. 
Die tägliche Futteraufnahme (TFM), die tägliche Besuchshäufigkeit (TBH) und die tägliche Besuchsdauer (TBD) wurden mit dem folgenden Modell analysiert:

$$
\mathrm{y}_{\mathrm{ijklm}}=\mu+\mathrm{FA}_{\mathrm{i}}+\mathrm{W}_{\mathrm{j}}+\mathrm{HJ}_{\mathrm{k}}+\mathrm{R}_{\mathrm{l}}+\mathrm{b} \times \mathrm{K}_{\mathrm{ijklmn}}+\mathrm{a}_{\mathrm{m}}+\mathrm{pu}_{\mathrm{m}}+\mathrm{e}_{\mathrm{ijklmno}}
$$

mit: $y_{\mathrm{ijklmno}}=$ Beobachtungswerte am n-ten Futtertag, $\mathrm{FA}_{\mathrm{i}}=$ fixer Effekt des i-ten Futterautomaten, $\mathrm{W}_{\mathrm{i}}=$ fixer Effekt der $\mathrm{j}$-ten Woche ( ${ }^{*}$ Station), $\mathrm{HJ}_{\mathrm{i}}=$ fixer Effekt des k-ten Herkunftsbetriebsjahres, $\mathrm{R}_{1}=$ fixer Effekt der 1-ten Rasse (ML, MLW), $b=$ linearer Regressionskoeffizient, $\mathrm{KM}_{\mathrm{ijklmn}}=$ interpolierte Körpermasse des Tieres am n-ten Futtertag (nur für Futteraufnahme), $\mathrm{a}_{\mathrm{m}}=$ additiv genetischer Effekt des Tieres i; $\mathrm{pu}_{\mathrm{m}}=$ permanenter Umwelteffekt des Tieres $\mathrm{i}, \mathrm{e}_{\mathrm{ijklmno}}=$ zufälliger Restfehler.

Dabei galt der Herkunftsbetrieb, dessen Einfluss noch in der Station nachwirken kann, als Umwelteffekt. Da sich die Effekte im Laufe der Zeit stark ändern können, wurden sie innerhalb von Jahren betrachtet. Im Thüringer Material erfolgte darüber hinaus eine Unterscheidung zwischen den Rassen Merinoland- und Merinolangwollschaf unterschieden.

Beim Wiederholbarkeitsmodell wurden alle Varianzen als fest und unabhängig von Alter oder Körpermasse angesehen.

\section{Ergebnisse und Diskussion}

\section{Heritabilitäten}

Für die Futteraufnahme und den Futteraufwand konnten mittlere bis hohe Heritabilitäten geschätzt werden (Tabelle 3).

Die Heritabilitäten der Futteraufnahme lagen mit 0,29 in Schöndorf und 0,58 in Grub in einem ähnlichen Bereich wie LEYMASTER et al. (2002) $\left(\mathrm{h}^{2}=0,25\right)$, FRANCOIS et al. $(2002)\left(h^{2}=0,43\right)$ oder SNOWDER und VAN VLECK $(2003)\left(h^{2}=0,39\right)$ an Schafen für alle Futterkomponenten ermittelten. Auch im Vergleich $\mathrm{zu}$ anderen Arbeiten an Rindern lagen die Werte aus Grub eher im hohen und die aus Schöndorf im niedrigen Bereich (NIEUWHOF et al., 1992; JENSEN et al., 1995; JAKOBSEN, 1995; MADSEN et al., 1995; FAN et al., 1995; WASSMUTH, 1999; POTTHAST, 1999; WASSMUTH und ALPS, 2000; MAC NEIL, 1991; ARCHER et al., 1997; ARTHUR et al., 2001; NKRUHMAH et al., 2007). Allerdings ist zu beachten, dass im Rinderbereich in der Regel mit Wiegetrögen, die die Erfassung der Kraft- und Grundfutteraufnahme ermöglichten, gearbeitet wurde. Die hier analysierten Daten bezogen im Gegensatz dazu lediglich die Kraftfuttermenge ein. Die Unterschiede zwischen den Stationen waren möglicherweise auf die unterschiedlichen Grundfutterarten und -qualitäten zurückzuführen. Für Mastleistungsmerkmale fanden KEFELGEN et al. (1998) ebenfalls ähnliche Differenzen zwischen verschiedenen Prüfstationen.

Für den in Deutschland üblichen Futteraufwand je Einheit Zuwachs ergaben sich prinzipiell ähnliche Schätzwerte wie für die Futteraufnahme. Die hohen genetischen Korrelationen zwischen diesen beiden Merkmalen machen deutlich, dass bei masseabhängiger Prüfung die Entscheidung für eines der beiden Merkmale zu nahezu der gleichen Zuchttierauswahl führen würde. Auch in den Untersuchungen von MAC NEIL (1991), FAN et al. (1995), ARCHER et al. (1997), ARTHUR et al. (2001) und NKRUHMAH et al. (2007) erreichten die Heritabilitäten des Futteraufwandes Größenordnungen, die für züchterische Maßnahmen ausreichten. Allerdings ist zu berücksichtigen, dass die Leistungsprüfung wie auch bei der hier vorgelegten 
Untersuchung gewichtsabhängig durchgeführt wurde. So fanden SNOWDER und VAN VLECK (2003) bei altersabhängiger Prüfung deutlich niedrigere Heritabilitäten für die Futterverwertung.

Die Schätzwerte der Heritabilität für die tägliche Futteraufnahme lagen mit 0,05 und 0,10 im niedrigen Bereich (Tabelle 3). Wegen der niedrigen Komponenten der permanenten Umwelt führen viele Messwiederholungen dennoch $\mathrm{zu}$ hohen Genauigkeiten. CAMMACK et al. (2005) schätzten eine Heritabilität von $\mathrm{h}^{2}=0,25$, GALLIVAN und SULLIVAN (1994) fanden einen Wert von 0,66 und die Studien von FRANCOIS et al. (2002) ergaben einen Wert von 0,43 für Mittelwerte der täglich aufgenommene Futtermenge. Werden etwa 30 Messungen unterstellt, entspricht dies dem Bereich der hier gefundenen Werte.

Allerdings lagen den genannten Studien nur niedrige Tierzahlen zugrunde.

Die Merkmale des Futteraufnahmeverhaltens (TBH, TBD) zeigten mittlere Heritabilitäten (Tabelle 3). Die geschätzte Heritabilität der täglichen Besuchshäufigkeit liegt mit 0,33 in Schöndorf und 0,49 in Grub für Einzelwerte erstaunlich hoch (Tabelle 3). CAMMACK et al. (2005) schätzten einen Wert von 0,35 für Mittelwerte der täglichen Besuchshäufigkeit.

Tabelle 3

Heritabilitäten $\left(\mathrm{h}^{2}\right)$ und Anteile der permanenten Umwelt (pU) (Standardfehler als Subskript) der Futteraufnahme (FAP), des Futteraufwandes (FAW), der täglichen Futteraufnahme (TFM), der täglichen Besuchshäufigkeit (TBH) und der täglichen Besuchsdauer (TBD) in Schöndorf und Grub

(Heritabilities and proportion of permanent environment [pU] [standard error as subscript] for feed intake [FAP], feed efficiency [FAW], daily feed intake [TFM], daily visits at the feeding rack [TBH] and daily eating time [TBD] in "Schöndorf" and "Grub")

\begin{tabular}{|c|c|c|c|c|}
\hline \multirow{2}{*}{ Merkmale } & \multicolumn{2}{|c|}{ Schöndorf } & \multicolumn{2}{|c|}{ Grub } \\
\hline & $\mathrm{h}^{2}$ & $\mathrm{pU}$ & $\mathrm{h}^{2}$ & $\mathrm{pU}$ \\
\hline$\overline{\text { FAP }}$ & $0,29_{0,05}$ & & $0,58_{0,08}$ & \\
\hline FAW & $0,32_{0,05}$ & & $0,57_{0,07}$ & \\
\hline TFM & $0,05_{0,01}$ & $0,08_{0,01}$ & $0,10_{0,01}$ & $0,02_{0,01}$ \\
\hline $\mathrm{TBH}$ & $0,33_{0,05}$ & $0.17_{0,04}$ & $0,49_{0,05}$ & $0,04_{0,04}$ \\
\hline TBD & $0,25_{0,04}$ & $0,21_{0,03}$ & $0,36_{0,06}$ & $0,08_{0,05}$ \\
\hline
\end{tabular}

\section{Genetische Korrelationen}

Die genetischen Korrelationen zwischen der Futteraufnahme und dem Fressverhalten zeigt Tabelle 4. So ergab sich zwischen der Futteraufnahme und dem Futteraufwand eine enge genetische Korrelation in beiden Stationen. Dies ist zum Teil auf die gewichtsabhängige Leistungsprüfung zurück zuführen. KENNEDY et al. (1993) äußerten Bedenken bei der Nutzung von Effizienzkriterien in der Zucht, da diese lediglich eine Funktion anderer Merkmale darstellen. Hieraus kann gefolgert werden, dass das Merkmal Futteraufnahme im Vordergrund der züchterischen Bemühungen stehen sollte.

Die täglich aufgenommene Futtermenge wies lediglich niedrige (Grub) bis mittlere (Schöndorf) genetische Korrelationen zu den Merkmalen Futteraufnahme bzw. Futteraufwand auf. Daher scheint eine gesonderte Betrachtung dieses Merkmals in der praktischen Zuchtarbeit angebracht.

Die genetischen Korrelationen zwischen den Verhaltensmerkmalen (TBH, TBD) und den Futteraufnahmemerkmalen (FAP, FAW, TFM) blieben insgesamt niedrig. Ähnliche Beobachtungen machten CAMMACK et al. (2005) bei Schafen und WASSMUTH und ALPS (2000) bei Rindern. Somit kann nicht vom Fressverhalten auf die aufgenommene Futtermenge geschlossen werden. 
Tabelle 4

Genetische Korrelationen (Standardfehler als Subskript) zwischen der Futteraufnahme (FAP), dem Futteraufwand (FAW), der täglichen Futteraufnahme (TFM), der täglichen Besuchshäufigkeit (TBH) und der täglichen Besuchsdauer (TBD) in Schöndorf und Grub

(Genetic correlations [standard error as subscript] between feed intake [FAP], feed efficiency [FAW], daily feed intake [TFM], daily visits at the feeding rack [TBH] and daily eating time [TBD] in "Schöndorf" and "Grub")

\begin{tabular}{lcccc}
\hline Merkmal & FAW & TFM & TBH & TBD \\
\hline $\begin{array}{l}\text { Schöndorf } \\
\text { FAP }\end{array}$ & & & $0,16_{0,03}$ & $0,08_{0,034}$ \\
FAW & $0,97_{0,01}$ & $0,64_{0,05}$ & $0,17_{0,04}$ & $0,09_{0,034}$ \\
TFM & & $0,62_{0,05}$ & $0,21_{0,12}$ & $0,03_{0,12}$ \\
TBH & & & $0,43_{0,08}$ \\
Grub & & & $0,05_{0,005}$ & $0,001_{0,04}$ \\
FAP & $1,00_{0,001}$ & $0,29_{0,05}$ & $0,03_{0,004}$ & $-0,004_{0,04}$ \\
FAW & & $0,28_{0,05}$ & $0,36_{0,10}$ & $0,23_{0,11}$ \\
TFM & & & $0,13_{0,03}$ \\
TBH & & & & \\
\hline
\end{tabular}

Schlussfolgerungen

Die Futteraufnahme im gesamten Prüfabschnitt weist eine züchterisch interessante Variabilität und Heritabilität auf.

Zwischen dem Futteraufwand und der Futteraufnahme im Prüfzeitraum konnte eine starke Abhängigkeit beobachtet werden.

Die Merkmale des Fressverhaltens weisen mittlere Heritabilitäten auf. Allerdings besitzen sie nur lose Beziehungen zur aufgenommenen Futtermenge.

Die Nutzung der täglichen Futteraufnahme für Zuchtwertschätzung und Selektion lässt keinen Vorteil gegenüber kumulierten Werten erwarten.

Die Literaturquellen und die Angaben zu den Autoren sind in der zweiten Mitteilung enthalten. 